\title{
Modified Dispersion Relations and Noncommutative Geometry lead to a finite Zero Point Energy
}

\author{
Remo Garattini \\ Università degli Studi di Bergamo, Facoltà di Ingegneria, \\ Viale Marconi 5, 24044 Dalmine (Bergamo) Italy and \\ I.N.F.N. - sezione di Milano, Milan, Italy. \\ E-mail:remo.garattini@unibg.it
}

\begin{abstract}
We compute Zero Point Energy in a spherically symmetric background with the help of the Wheeler-DeWitt equation. This last one is regarded as a Sturm-Liouville problem with the cosmological constant considered as the associated eigenvalue. The graviton contribution, at one loop is extracted wit the help of a variational approach together with Gaussian trial functionals. The divergences handled with a zeta function regularization are compared with the results obtained using a Noncommutative Geometry (NCG) and Modified Dispersion Relations (MDR). In both NCG and MDR no renormalization scheme is necessary to remove infinities in contrast to what happens in conventional approaches.
\end{abstract}

Keywords: Cosmological Constant, Quantum Cosmology, Quantum Gravity, Noncommutative Geometry, Modified Dispersion Relations

PACS: 04.60.-m, 04.62+v, 05.10.Cc

\section{INTRODUCTION}

Quantum Gravity should be the fusion between Quantum Mechanics and General Relativity. When they are disconnected these theories work pretty good in their respective domains. However, when one tries to merge them in a single theory, one discovers that this last one is non renormalizable and therefore its predictive power is lost. Recently, Hořava proposed a modification of Einstein gravity motivated by the Lifshitz theory in solid state physics[1][2]. Such modification allows the theory to be power-counting ultraviolet (UV)-renormalizable and should recover general relativity in the infrared (IR) limit. Nevertheless Hořava-Lifshitz (HL) theory is non-covariant, at least in its original formulation ${ }^{1}$. Indeed, in this approach space and time exhibit Lifshitz scale invariance of the form

$$
t \rightarrow \ell^{z} t \text { and } x^{i} \rightarrow \ell x^{i}
$$

with $z \geq 1 . z$ is called the dynamical critical exponent. The breaking of the 4D diffeomorphism invariance allows a different treatment of the kinetic and potential terms for the metric: from one side the kinetic term is quadratic in time derivatives of the metric, from the other side the potential has high-order space derivatives. In particular the UV behav-

\footnotetext{
${ }^{1}$ See Ref.[3]. for recent progress to obtain a covariant theory.
} 
ior is dominated by the square of the Cotton tensor of the $3 D$ geometry by means of a $k^{6}$ contribution to the propagator leading to a renormalizable power-counting theory. The original HL theory is based on two assumptions - detailed balance and projectability[4]. The projectability condition is a weak version of the invariance with respect to time reparametrization and therefore to the Wheeler-DeWitt (WDW) equation[5]. In this contribution, we do not want to discuss the appealing properties of the HL theory, but rather, we would like to investigate the original WDW equation with a look to possible deformations induced by the spacetime itself. Therefore no matter fields will be included in such a discussion. We have in mind two interesting cases: Non Commutative Geometry and Rainbow's Gravity. Both of these cases are modifications of the original space time structure. For this reason we expect some interesting consequences on the computation of some observables. In this paper, we are interested to see what are the effects of such distortions on Zero Point Energy (ZPE). A partial promising answer has been obtained in Ref.[6], where the UV divergences ogf a Schwarzschild black hole are kept under control by an appropriate choice of the Rainbow's metric. Units in which $\hbar=c=k=1$ are used throughout the paper.

\section{THE WDW EQUATION}

The WDW equation was originally introduced by Bryce DeWitt as an attempt to quantize General Relativity in a Hamiltonian formulation. It is described by

$$
\mathscr{H} \Psi=\left[(2 \kappa) G_{i j k l} \pi^{i j} \pi^{k l}-\frac{\sqrt{g}}{2 \kappa}\left({ }^{3} R-2 \Lambda\right)\right] \Psi=0
$$

and it represents the quantum version of the classical constraint which guarantees the invariance under time reparametrization. $G_{i j k l}$ is the super-metric, $\pi^{i j}$ is the supermomentum, ${ }^{3} R$ is the scalar curvature in three dimensions and $\Lambda$ is the cosmological constant, while $\kappa=8 \pi G$ with $G$ the Newton's constant. In this way, the WDW equation is written in its most general form. The main reason to use such an equation to discuss renormalization problems is related to the possibility of formally re-writing the WDW equation as an expectation value computation ${ }^{2}$ [7]. Indeed, if we multiply Eq.(2) by $\Psi^{*}\left[g_{i j}\right]$ and functionally integrate over the three spatial metric $g_{i j}$ we find

$$
\frac{1}{V} \frac{\int \mathscr{D}\left[g_{i j}\right] \Psi^{*}\left[g_{i j}\right] \int_{\Sigma} d^{3} x \hat{\Lambda}_{\Sigma} \Psi\left[g_{i j}\right]}{\int \mathscr{D}\left[g_{i j}\right] \Psi^{*}\left[g_{i j}\right] \Psi\left[g_{i j}\right]}=\frac{1}{V} \frac{\left\langle\Psi\left|\int_{\Sigma} d^{3} x \hat{\Lambda}_{\Sigma}\right| \Psi\right\rangle}{\langle\Psi \mid \Psi\rangle}=-\frac{\Lambda}{\kappa} .
$$

In Eq.(3) we have also integrated over the hypersurface $\Sigma$ and we have defined

$$
V=\int_{\Sigma} d^{3} x \sqrt{g}
$$

as the volume of the hypersurface $\Sigma$ with

$$
\hat{\Lambda}_{\Sigma}=(2 \kappa) G_{i j k l} \pi^{i j} \pi^{k l}-\sqrt{g}^{3} R /(2 \kappa) .
$$

\footnotetext{
${ }^{2}$ See also Ref.[8] for an application of the method to a $f(R)$ theory.
} 
In this form, Eq.(3) can be used to compute ZPE provided that $\Lambda / \kappa$ be considered as an eigenvalue of $\hat{\Lambda}_{\Sigma}$. In particular, Eq.(3) represents the Sturm-Liouville problem associated with the cosmological constant. To solve Eq.(3) is a quite impossible task. Therefore, we are oriented to use a variational approach with trial wave functionals. The related boundary conditions are dictated by the choice of the trial wave functionals which, in our case are of the Gaussian type. Different types of wave functionals correspond to different boundary conditions. The choice of a Gaussian wave functional is justified by the fact that ZPE should be described by a good candidate of the "vacuum state". To fix ideas, we choose the following form of the metric

$$
d s^{2}=-N^{2}(r) d t^{2}+\frac{d r^{2}}{1-\frac{b(r)}{r}}+r^{2}\left(d \theta^{2}+\sin ^{2} \theta d \phi^{2}\right)
$$

where $b(r)$ is subject to the only condition $b\left(r_{t}\right)=r_{t}$. As a first step, we decompose the gravitational perturbation in such a way to obtain the graviton contribution enclosed in Eq.(3).

\section{Extracting the graviton contribution}

We can gain more information if we consider $g_{i j}=\bar{g}_{i j}+h_{i j}$, where $\bar{g}_{i j}$ is the background metric and $h_{i j}$ is a quantum fluctuation around the background. Thus Eq.(3) can be expanded in terms of $h_{i j}$. Since the kinetic part of $\hat{\Lambda}_{\Sigma}$ is quadratic in the momenta, we only need to expand the three-scalar curvature $\int d^{3} x \sqrt{g}^{3} R$ up to the quadratic order. However, to proceed with the computation, we also need an orthogonal decomposition on the tangent space of 3-metric deformations [9, 10]:

$$
h_{i j}=\frac{1}{3}(\sigma+2 \nabla \cdot \xi) g_{i j}+(L \xi)_{i j}+h_{i j}^{\perp} .
$$

The operator $L$ maps $\xi_{i}$ into symmetric tracefree tensors

$$
(L \xi)_{i j}=\nabla_{i} \xi_{j}+\nabla_{j} \xi_{i}-\frac{2}{3} g_{i j}(\nabla \cdot \xi),
$$

$h_{i j}^{\perp}$ is the traceless-transverse component of the perturbation (TT), namely $g^{i j} h_{i j}^{\perp}=0$, $\nabla^{i} h_{i j}^{\perp}=0$ and $h$ is the trace of $h_{i j}$. It is immediate to recognize that the trace element $\sigma=h-2(\nabla \cdot \xi)$ is gauge invariant. If we perform the same decomposition also on the momentum $\pi^{i j}$, up to second order Eq.(3) becomes

$$
\frac{1}{V} \frac{\left\langle\Psi\left|\int_{\Sigma} d^{3} x\left[\hat{\Lambda}_{\Sigma}^{\perp}+\hat{\Lambda}_{\Sigma}^{\xi}+\hat{\Lambda}_{\Sigma}^{\sigma}\right]^{(2)}\right| \Psi\right\rangle}{\langle\Psi \mid \Psi\rangle}=-\frac{\Lambda}{\kappa} .
$$

Concerning the measure appearing in Eq.(3), we have to note that the decomposition (7) induces the following transformation on the functional measure $\mathscr{D} h_{i j} \rightarrow \mathscr{D} h_{i j}^{\perp} \mathscr{D} \xi_{i} \mathscr{D} \sigma J_{1}$, 
where the Jacobian related to the gauge vector variable $\xi_{i}$ is

$$
J_{1}=\left[\operatorname{det}\left(\triangle g^{i j}+\frac{1}{3} \nabla^{i} \nabla^{j}-R^{i j}\right)\right]^{\frac{1}{2}} .
$$

This is nothing but the famous Faddev-Popov determinant. It becomes more transparent if $\xi_{a}$ is further decomposed into a transverse part $\xi_{a}^{T}$ with $\nabla^{a} \xi_{a}^{T}=0$ and a longitudinal part $\xi_{a}^{\|}$with $\xi_{a}^{\|}=\nabla_{a} \psi$, then $J_{1}$ can be expressed by an upper triangular matrix for certain backgrounds (e.g. Schwarzschild in three dimensions). It is immediate to recognize that for an Einstein space in any dimension, cross terms vanish and $J_{1}$ can be expressed by a block diagonal matrix. Since $\operatorname{det} A B=\operatorname{det} A \operatorname{det} B$, the functional measure $\mathscr{D} h_{i j}$ factorizes into

$$
\mathscr{D} h_{i j}=\left(\operatorname{det} \triangle_{V}^{T}\right)^{\frac{1}{2}}\left(\operatorname{det}\left[\frac{2}{3} \triangle^{2}+\nabla_{i} R^{i j} \nabla_{j}\right]\right)^{\frac{1}{2}} \mathscr{D} h_{i j}^{\perp} \mathscr{D} \xi^{T} \mathscr{D} \psi
$$

with $\left(\triangle_{V}^{i j}\right)^{T}=\triangle g^{i j}-R^{i j}$ acting on transverse vectors, which is the Faddeev-Popov determinant. In writing the functional measure $\mathscr{D} h_{i j}$, we have here ignored the appearance of a multiplicative anomaly[11]. Thus the inner product can be written as

$$
\int \mathscr{D} \rho \Psi^{*}\left[h_{i j}^{\perp}\right] \Psi^{*}\left[\xi^{T}\right] \Psi^{*}[\sigma] \Psi\left[h_{i j}^{\perp}\right] \Psi\left[\xi^{T}\right] \Psi[\sigma]
$$

where

$$
\mathscr{D} \rho=\mathscr{D} h_{i j}^{\perp} \mathscr{D} \xi^{T} \mathscr{D} \sigma\left(\operatorname{det} \triangle_{V}^{T}\right)^{\frac{1}{2}}\left(\operatorname{det}\left[\frac{2}{3} \triangle^{2}+\nabla_{i} R^{i j} \nabla_{j}\right]\right)^{\frac{1}{2}} .
$$

Nevertheless, since there is no interaction between ghost fields and the other components of the perturbation at this level of approximation, the Jacobian appearing in the numerator and in the denominator simplify. The reason can be found in terms of connected and disconnected terms. The disconnected terms appear in the Faddeev-Popov determinant and these ones are not linked by the Gaussian integration. This means that disconnected terms in the numerator and the same ones appearing in the denominator cancel out. Therefore, Eq.(9) factorizes into three pieces. The piece containing $\hat{\Lambda}_{\Sigma}^{\perp}$ is the contribution of the transverse-traceless tensors (TT): essentially is the graviton contribution representing true physical degrees of freedom. Regarding the vector term $\hat{\Lambda}_{\Sigma}^{T}$, we observe that under the action of infinitesimal diffeomorphism generated by a vector field $\varepsilon_{i}$, the components of (7) transform as follows[9]

$$
\xi_{j} \longrightarrow \xi_{j}+\varepsilon_{j}, \quad h \longrightarrow h+2 \nabla \cdot \xi, \quad h_{i j}^{\perp} \longrightarrow h_{i j}^{\perp}
$$

The Killing vectors satisfying the condition $\nabla_{i} \xi_{j}+\nabla_{j} \xi_{i}=0$, do not change $h_{i j}$, and thus should be excluded from the gauge group. All other diffeomorphisms act on $h_{i j}$ nontrivially. We need to fix the residual gauge freedom on the vector $\xi_{i}$. The simplest choice is $\xi_{i}=0$. This new gauge fixing produces the same Faddeev-Popov determinant connected to the Jacobian $J_{1}$ and therefore will not contribute to the final value. We are 
left with

$$
\frac{1}{V} \frac{\left\langle\Psi^{\perp}\left|\int_{\Sigma} d^{3} x\left[\hat{\Lambda}_{\Sigma}^{\perp}\right]^{(2)}\right| \Psi^{\perp}\right\rangle}{\left\langle\Psi^{\perp} \mid \Psi^{\perp}\right\rangle}+\frac{1}{V} \frac{\left\langle\Psi^{\sigma}\left|\int_{\Sigma} d^{3} x\left[\hat{\Lambda}_{\Sigma}^{\sigma}\right]^{(2)}\right| \Psi^{\sigma}\right\rangle}{\left\langle\Psi^{\sigma} \mid \Psi^{\sigma}\right\rangle}=-\frac{\Lambda^{\perp}}{\kappa}-\frac{\Lambda^{\sigma}}{\kappa} .
$$

Note that in the expansion of $\int_{\Sigma} d^{3} x \sqrt{g} R$ to second order, a coupling term between the TT component and scalar one remains. However, the Gaussian integration does not allow such a mixing which has to be introduced with an appropriate wave functional. Extracting the TT tensor contribution from Eq.(3) approximated to second order in perturbation of the spatial part of the metric into a background term $\bar{g}_{i j}$, and a perturbation $h_{i j}$, we get

$$
\hat{\Lambda}_{\Sigma}^{\perp}=\frac{1}{4 V} \int_{\Sigma} d^{3} x \sqrt{\bar{g}} G^{i j k l}\left[(2 \kappa) K^{-1 \perp}(x, x)_{i j k l}+\frac{1}{(2 \kappa)}\left(\tilde{\triangle}_{L}\right)_{j}^{a} K^{\perp}(x, x)_{i a k l}\right],
$$

where

$$
\left(\tilde{\triangle}_{L} h^{\perp}\right)_{i j}=\left(\triangle_{L} h^{\perp}\right)_{i j}-4 R_{i}^{k} h_{k j}^{\perp}+{ }^{3} R h_{i j}^{\perp}
$$

is the modified Lichnerowicz operator and $\triangle_{L}$ is the Lichnerowicz operator defined by

$$
\left(\triangle_{L} h\right)_{i j}=\triangle h_{i j}-2 R_{i k j l} h^{k l}+R_{i k} h_{j}^{k}+R_{j k} h_{i}^{k} \quad \triangle=-\nabla^{a} \nabla_{a} .
$$

$G^{i j k l}$ represents the inverse DeWitt metric and all indices run from one to three. Note that the term $-4 R_{i}^{k} h_{k j}^{\perp}+{ }^{3} R h_{i j}^{\perp}$ disappears in four dimensions. The propagator $K^{\perp}(x, x)_{i a k l}$ can be represented as

$$
K^{\perp}(\vec{x}, \vec{y})_{i a k l}=\sum_{\tau} \frac{h_{i a}^{(\tau) \perp}(\vec{x}) h_{k l}^{(\tau) \perp}(\vec{y})}{2 \lambda(\tau)},
$$

where $h_{i a}^{(\tau) \perp}(\vec{x})$ are the eigenfunctions of $\tilde{\triangle}_{L} \cdot \tau$ denotes a complete set of indices and $\lambda(\tau)$ are a set of variational parameters to be determined by the minimization of Eq.(16). The expectation value of $\hat{\Lambda}_{\Sigma}^{\perp}$ is easily obtained by inserting the form of the propagator into Eq.(16) and minimizing with respect to the variational function $\lambda(\tau)$. Thus the total one loop energy density for TT tensors becomes

$$
\frac{\Lambda}{8 \pi G}=-\frac{1}{2} \sum_{\tau}\left[\sqrt{\omega_{1}^{2}(\tau)}+\sqrt{\omega_{2}^{2}(\tau)}\right] .
$$

The above expression makes sense only for $\omega_{i}^{2}(\tau)>0$, where $\omega_{i}$ are the eigenvalues of $\tilde{\triangle}_{L}$. In the next section, we will explicitly evaluate Eq.(20) for a background of spherically symmetric type.

\section{ONE LOOP ENERGY DENSITY: CONVENTIONAL REGULARIZATION AND RENORMALIZATION}

The reference metric (6) can be cast into the following form

$$
d s^{2}=-N^{2}(r(x)) d t^{2}+d x^{2}+r^{2}(x)\left(d \theta^{2}+\sin ^{2} \theta d \phi^{2}\right),
$$


where

$$
d x= \pm \frac{d r}{\sqrt{1-\frac{b(r)}{r}}}
$$

Specific examples are

$$
b(r)=\frac{\Lambda_{d S}}{3} r^{3} ; \quad b(r)=-\frac{\Lambda_{A d S}}{3} r^{3} \quad \text { and } \quad b(r)=2 M G .
$$

However, we would like to maintain the form of the line element (21) as general as possible. With the help of Regge and Wheeler representation[12], the Lichnerowicz operator $\left(\tilde{\triangle}_{L} h^{\perp}\right)_{i j}$ can be reduced to

$$
\left[-\frac{d^{2}}{d x^{2}}+\frac{l(l+1)}{r^{2}}+m_{i}^{2}(r)\right] f_{i}(x)=\omega_{i, l}^{2} f_{i}(x) \quad i=1,2 \quad,
$$

where we have used reduced fields of the form $f_{i}(x)=F_{i}(x) / r$ and where we have defined two r-dependent effective masses $m_{1}^{2}(r)$ and $m_{2}^{2}(r)$

$$
\left\{\begin{array}{l}
m_{1}^{2}(r)=\frac{6}{r^{2}}\left(1-\frac{b(r)}{r}\right)+\frac{3}{2 r^{2}} b^{\prime}(r)-\frac{3}{2 r^{3}} b(r) \\
m_{2}^{2}(r)=\frac{6}{r^{2}}\left(1-\frac{b(r)}{r}\right)+\frac{1}{2 r^{2}} b^{\prime}(r)+\frac{3}{2 r^{3}} b(r)
\end{array}(r \equiv r(x)) .\right.
$$

In order to use the W.K.B. method considered by 't Hooft in the brick wall problem[13], from Eq.(24) we can extract two r-dependent radial wave numbers

$$
k_{i}^{2}\left(r, l, \omega_{i, n l}\right)=\omega_{i, n l}^{2}-\frac{l(l+1)}{r^{2}}-m_{i}^{2}(r) \quad i=1,2
$$

Then the counting of the number of modes with frequency less than $\omega_{i}$ is given approximately by

$$
\tilde{g}\left(\omega_{i}\right)=\int_{0}^{l_{\max }} v_{i}\left(l, \omega_{i}\right)(2 l+1) d l .
$$

$v_{i}\left(l, \omega_{i}\right)$ is the number of nodes in the mode with $\left(l, \omega_{i}\right)$, such that $(r \equiv r(x))$

$$
v_{i}\left(l, \omega_{i}\right)=\frac{1}{\pi} \int_{-\infty}^{+\infty} d x \sqrt{k_{i}^{2}\left(r, l, \omega_{i}\right)} .
$$

Here it is understood that the integration with respect to $x$ and $l_{\max }$ is taken over those values which satisfy $k_{i}^{2}\left(r, l, \omega_{i}\right) \geq 0$. With the help of Eqs. $(27,28)$, Eq. (20) becomes

$$
\frac{\Lambda}{8 \pi G}=-\frac{1}{\pi} \sum_{i=1}^{2} \int_{0}^{+\infty} \omega_{i} \frac{d \tilde{g}\left(\omega_{i}\right)}{d \omega_{i}} d \omega_{i}
$$


This is the one loop graviton contribution to the induced cosmological constant. The explicit evaluation of Eq.(29) gives

$$
\frac{\Lambda}{8 \pi G}=\rho_{1}+\rho_{2}=-\frac{1}{4 \pi^{2}} \sum_{i=1}^{2} \int_{\sqrt{m_{i}^{2}(r)}}^{+\infty} \omega_{i}^{2} \sqrt{\omega_{i}^{2}-m_{i}^{2}(r)} d \omega_{i}
$$

where we have included an additional $4 \pi$ coming from the angular integration. The use of the zeta function regularization method to compute the energy densities $\rho_{1}$ and $\rho_{2}$ leads to

$$
\rho_{i}(\varepsilon)=\frac{m_{i}^{4}(r)}{64 \pi^{2}}\left[\frac{1}{\varepsilon}+\ln \left(\frac{4 \mu^{2}}{m_{i}^{2}(r) \sqrt{e}}\right)\right] \quad i=1,2,
$$

where we have introduced the additional mass parameter $\mu$ in order to restore the correct dimension for the regularized quantities. Such an arbitrary mass scale emerges unavoidably in any regularization scheme. The renormalization is performed via the absorption of the divergent part into the re-definition of a bare classical quantity. Here we have two possible choices: the induced cosmological constant $\Lambda$ or the gravitational Newton constant $G$. If we decide to absorb the divergence with the help of the cosmological constant $\Lambda$, we have to separate it into a bare cosmological constant $\Lambda_{0}$ and a divergent quantity $\Lambda^{d i v}$, where

$$
\Lambda^{d i v}=\frac{G m_{0}^{4}(r)}{\varepsilon 32 \pi^{2}},
$$

and the remaining finite value for the cosmological constant reads

$$
\frac{\Lambda_{0}}{8 \pi G}=\left(\rho_{1}(\mu)+\rho_{2}(\mu)\right)=\rho_{e f f}^{T T}(\mu, r)=\frac{m_{0}^{4}(r)}{32 \pi^{2}} \ln \left(\frac{4 \mu^{2}}{m_{0}^{2}(r) \sqrt{e}}\right) .
$$

\section{THE EXAMPLE OF NON COMMUTATIVE THEORIES}

Non Commutative theories provide a powerful method to naturally regularize divergent integrals appearing in Eq.(30). Basically, the number of states is modified in the following way[16]

$$
d n=\frac{d^{3} x d^{3} k}{(2 \pi)^{3}} \Longrightarrow d n_{i}=\frac{d^{3} x d^{3} k}{(2 \pi)^{3}} \exp \left(-\frac{\theta}{4}\left(\omega_{i, n l}^{2}-m_{i}^{2}(r)\right)\right), \quad i=1,2 .
$$

This deformation corresponds to an effective cut off on the background geometry (21). The UV cut off is triggered only by higher momenta modes $\gtrsim 1 / \sqrt{\theta}$ which propagate over the background geometry. The virtue of this kind of deformation is its exponential damping profile, which encodes an intrinsic nonlocal character into fields $f_{i}(x)$. Plugging (28) into (27) and taking account of (34), the number of modes with frequency less than $\omega_{i}, i=1,2$ is given by

$$
\tilde{g}\left(\omega_{i}\right)=\frac{1}{\pi} \int_{-\infty}^{+\infty} d x \int_{0}^{l_{\max }}(2 l+1) \sqrt{\omega_{i, n l}^{2}-\frac{l(l+1)}{r^{2}}-m_{i}^{2}(r)} \exp \left(-\frac{\theta}{4} k_{i}^{2}\right) d l
$$


and the induced cosmological constant becomes

$$
\begin{aligned}
\frac{\Lambda}{8 \pi G} & =\frac{1}{6 \pi^{2}}\left[\int_{\sqrt{m_{0}^{2}(r)}}^{+\infty} \sqrt{\left(\omega^{2}-m_{0}^{2}(r)\right)^{3}} e^{-\frac{\theta}{4}\left(\omega^{2}-m_{0}^{2}(r)\right)} d \omega\right. \\
& \left.+\int_{0}^{+\infty} \sqrt{\left(\omega^{2}+m_{0}^{2}(r)\right)^{3}} e^{-\frac{\theta}{4}\left(\omega^{2}+m_{0}^{2}(r)\right)} d \omega\right],
\end{aligned}
$$

where an integration by parts in Eq.(29) has been done. By further developing the calculations we find

$$
\frac{\Lambda}{8 \pi G}=\frac{1}{12 \pi^{2}}\left(\frac{4}{\theta}\right)^{2}\left(x \cosh \left(\frac{x}{2}\right)-x^{2} \sinh \left(\frac{x}{2}\right)\right) K_{1}\left(\frac{x}{2}\right)+x^{2} \cosh \left(\frac{x}{2}\right) K_{0}\left(\frac{x}{2}\right)
$$

where $K_{0}(x)$ and $K_{1}(x)$ are the modified Bessel function and

$$
x=\frac{m_{0}^{2}(r) \theta}{4}
$$

The asymptotic properties of (37) show that the one loop contribution is everywhere regular. Indeed, we find that when $x \rightarrow+\infty$,

$$
\frac{\Lambda}{8 \pi G} \simeq \frac{1}{6 \pi^{2} \theta^{2}} \sqrt{\frac{\pi}{x}}\left[3+\left(8 x^{2}+6 x+3\right) \exp (-x)\right] \rightarrow 0
$$

Conversely, when $x \rightarrow 0$, we obtain

$$
\frac{\Lambda}{8 \pi G} \simeq \frac{4}{3 \pi^{2} \theta^{2}}\left[2-\left(\frac{7}{8}+\frac{3}{4} \ln \left(\frac{x}{4}\right)+\frac{3}{4} \gamma\right) x^{2}\right] \rightarrow \frac{8}{3 \pi^{2} \theta^{2}}
$$

a finite value for $\Lambda$. Note that expression (37) can be used when the background satisfies the relation

$$
m_{0}^{2}(r)=m_{1}^{2}(r)=-m_{2}^{2}(r) .
$$

Examples of metrics satisfying relation (41) are the Schwarzschild, Schwarzschild-de Sitter (SdS) and Schwarzschild-Anti de Sitter (SAdS) backgrounds ${ }^{3}$. The other interesting cases, namely de Sitter and Anti-de Sitter are described by

$$
m_{1}^{2}(r)=m_{2}^{2}(r)=m_{0}^{2}(r)
$$

leading to

$$
\frac{\Lambda}{8 \pi G}=\frac{1}{3 \pi^{2}}\left[\int_{\sqrt{m_{0}^{2}(r)}}^{+\infty} \sqrt{\left(\omega^{2}-m_{0}^{2}(r)\right)^{3}} e^{-\frac{\theta}{4}\left(\omega^{2}-m_{0}^{2}(r)\right)} d \omega\right]
$$

\footnotetext{
${ }^{3}$ Usually for such geometries, relation (41) is satisfied in a region close to the throat.
} 


$$
=\frac{1}{6 \pi^{2}}\left(\frac{4}{\theta}\right)^{2}\left(\frac{1}{2} y(1-y) K_{1}\left(\frac{y}{2}\right)+\frac{1}{2} y^{2} K_{0}\left(\frac{y}{2}\right)\right) \exp \left(\frac{y}{2}\right) .
$$

The asymptotic expansion of Eq.(43) leads to

$$
\frac{\Lambda}{8 \pi G} \simeq \frac{1}{6 \pi^{2}}\left(\frac{4}{\theta}\right)^{2} \frac{3}{8} \sqrt{\frac{\pi}{x}} \rightarrow 0,
$$

when $x \rightarrow \infty$. On the other hand, when $x \rightarrow 0$, one gets

$$
\frac{\Lambda}{8 \pi G} \simeq \frac{1}{6 \pi^{2}}\left(\frac{4}{\theta}\right)^{2}\left[1-\frac{x}{2}+\left(-\frac{7}{16}-\frac{3}{8} \ln \left(\frac{x}{4}\right)-\frac{3}{8} \gamma\right) x^{2}\right] \rightarrow \frac{8}{3 \pi^{2} \theta^{2}}
$$

i.e. a finite value of the cosmological term.

\section{RAINBOW'S GRAVITY AT WORK}

In recent years, there has been a proposal on how the fundamental aspects of special relativity can be modified at very high energies. This modification has been named Doubly Special Relativity (DSR)[17]. One of its effects is that the usual dispersion relation of a massive particle of mass $m$ is modified into the following expression

$$
E^{2} g_{1}^{2}\left(E / E_{P l}\right)-p^{2} g_{2}^{2}\left(E / E_{P l}\right)=m^{2},
$$

where $g_{1}\left(E / E_{P l}\right)$ and $g_{2}\left(E / E_{P l}\right)$ are two functions which have the following property

$$
\lim _{E / E_{P l} \rightarrow 0} g_{1}\left(E / E_{P l}\right)=1 \quad \text { and } \quad \lim _{E / E_{P l} \rightarrow 0} g_{2}\left(E / E_{P l}\right)=1 .
$$

Thus, the usual dispersion relation is recovered at low energies. This simple assumption has a deep impact also when the background is curved. For example, if we examine the Schwarzschild metric, the analysis of Magueijo and Smolin[18] shows that the energymomentum tensor and the Einstein equations are replaced by a one parameter family of equations

$$
G_{\mu \nu}(E)=8 \pi G(E) T_{\mu \nu}(E)+g_{\mu \nu} \Lambda(E),
$$

where $G(E)$ is an energy dependent Newton's constant, defined so that $G(0)$ is the physical Newton's constant. Similarly we have an energy dependent cosmological constant $\Lambda(E)$. A solution of the modified Einstein's Field Equations for a metric of the form (6) is represented by a "rainbow metric" whose line element reads

$$
d s^{2}=-\frac{N^{2}(r) d t^{2}}{g_{1}^{2}(E)}+\frac{d r^{2}}{\left(1-\frac{b(r)}{r}\right) g_{2}^{2}(E)}+\frac{r^{2}}{g_{2}^{2}(E)}\left(d \theta^{2}+\sin ^{2} \theta d \phi^{2}\right) .
$$

We expect the functions $g_{1}\left(E / E_{P l}\right)$ and $g_{2}\left(E / E_{P l}\right)$ modify the UV behavior in the same way as GUP and Noncommutative geometry do, respectively. In presence of Rainbow's 
Gravity, we find that Eq. $(2)^{4}$ becomes

$$
\tilde{\mathscr{H}} \Psi=\left[\frac{g_{1}^{2}(E)}{g_{2}^{3}(E)} \tilde{G}_{i j k l} \tilde{\pi}^{i j} \tilde{\pi}^{k l}-\frac{\sqrt{\tilde{g}}}{2 \kappa g_{2}(E)}\left(\tilde{R}-\frac{2 \Lambda_{c}}{g_{2}^{2}(E)}\right)\right] \Psi=0
$$

and, consequently Eq.(3) changes into

$$
\frac{g_{2}^{3}(E)}{\tilde{V}} \frac{\left\langle\Psi\left|\int_{\Sigma} d^{3} x \tilde{\Lambda}_{\Sigma}\right| \Psi\right\rangle}{\langle\Psi \mid \Psi\rangle}=-\frac{\Lambda}{\kappa}
$$

where

$$
\tilde{\Lambda}_{\Sigma}=(2 \kappa) \frac{g_{1}^{2}(E)}{g_{2}^{3}(E)} \tilde{G}_{i j k l} \tilde{\pi}^{i j} \tilde{\pi}^{k l}-\frac{\sqrt{\tilde{g}} \tilde{R}}{(2 \kappa) g_{2}(E)} .
$$

Of course, Eqs. $(50,51)$ and (52) reduce to the ordinary Eqs. $(2,3)$ and (5) when $E / E_{P l} \rightarrow 0$. By repeating the procedure of subsection, we find that the TT tensor contribution of Eq. (51) is

$\hat{\Lambda}_{\Sigma}^{\perp}=\frac{g_{2}^{3}(E)}{4 \tilde{V}} \int_{\Sigma} d^{3} x \sqrt{\tilde{\bar{g}}} \tilde{G}^{i j k l}\left[(2 \kappa) \frac{g_{1}^{2}(E)}{g_{2}^{3}(E)} \tilde{K}^{-1 \perp}(x, x)_{i j k l}+\frac{1}{(2 \kappa) g_{2}(E)}\left(\tilde{\triangle}_{L}^{m} \tilde{K}^{\perp}(x, x)\right)_{i j k l}\right]$

and the total one loop energy density becomes

$$
\frac{\Lambda}{8 \pi G}=-\frac{1}{2} \sum_{\tau} g_{1}(E) g_{2}(E)\left[\sqrt{E_{1}^{2}(\tau)}+\sqrt{E_{2}^{2}(\tau)}\right] .
$$

Since Eq.(24) is modified by the "rainbow metric", the two r-dependent radial wave numbers (26) become

$$
k_{i}^{2}\left(r, l, \omega_{i, n l}\right)=\frac{E_{i, n l}^{2}}{g_{2}^{2}(E)}-\frac{l(l+1)}{r^{2}}-m_{i}^{2}(r) \quad i=1,2
$$

and with the help of Eqs. $(27,28)$, Eq. (20) reduces to

$$
\frac{\Lambda}{8 \pi G}=-\frac{1}{3 \pi^{2}} \sum_{i=1}^{2} \int_{E^{*}}^{+\infty} E_{i} g_{1}(E) g_{2}(E) \frac{d}{d E_{i}} \sqrt{\left(\frac{E_{i}^{2}}{g_{2}^{2}(E)}-m_{i}^{2}(r)\right)^{3}} d E_{i}
$$

where $E^{*}$ is the value which annihilates the argument of the root. In the previous equation we have assumed that the effective mass does not depend on the energy $E$. To further proceed, we choose a form of $g_{1}\left(E / E_{P}\right)$ and $g_{2}\left(E / E_{P}\right)$ which allows a comparison with the results obtained with a Noncommutative geometry computation expressed by Eq.(36). If we fix

$$
g_{1}\left(E / E_{P}\right)=\exp \left(-\alpha \frac{E^{2}}{E_{P}^{2}}\right) \quad \text { and } \quad g_{2}\left(E / E_{P}\right)=1,
$$

\footnotetext{
${ }^{4}$ Details of the calculation related to the whole section can be found in Ref.[19]
} 
with $\alpha>0$, Eq.(56) becomes

$$
\begin{aligned}
\frac{\Lambda}{8 \pi G}= & -\frac{1}{\pi^{2}}\left[\int_{\sqrt{m_{0}^{2}(r)}}^{+\infty} E^{2} \exp \left(-\alpha \frac{E^{2}}{E_{P}^{2}}\right) \sqrt{\left(E^{2}-m_{0}^{2}(r)\right)} d E\right. \\
& \left.+\int_{0}^{+\infty} E^{2} \exp \left(-\alpha \frac{E^{2}}{E_{P}^{2}}\right) \sqrt{\left(E^{2}+m_{0}^{2}(r)\right)} d E\right],
\end{aligned}
$$

where we have used relation (41). Nevertheless we have to observe that even if the final result is finite and background dependent, the induced cosmological constant computed with the choice (57) will be always negative. When we compare this result with that obtained in Noncommutative geometry, namely Eq.(36), we find that the negativity is principally due the Rainbow's functions that do not completely enter in the counting of nodes like in expression (35). Therefore the pure "Gaussian" choice (57) can not give a positive induced cosmological constant. We are thus led to choose

$$
g_{1}\left(E / E_{P}\right)=\left(1+\beta \frac{E}{E_{P}}\right) \exp \left(-\alpha \frac{E^{2}}{E_{P}^{2}}\right) \quad \text { and } \quad g_{2}\left(E / E_{P}\right)=1,
$$

with $\alpha>0$ and $\beta \in \mathbb{R}$. Plugging parametrization (57) into expression (56), one gets

$$
\begin{gathered}
\Lambda \\
8 \pi G \\
=-\frac{E_{P}^{4}}{4 \pi^{2}}\left[\frac{x^{2}}{\alpha} \cosh \left(\frac{\alpha x^{2}}{2}\right) K_{1}\left(\frac{\alpha x^{2}}{2}\right)\right. \\
\left.-\beta\left(\frac{3 x}{2 \alpha^{2}}-\frac{x^{2} \sqrt{\pi}}{\alpha^{\frac{3}{2}}} \sinh \left(\alpha x^{2}\right)+\frac{3 \sqrt{\pi}}{2 \alpha^{\frac{5}{2}}} \cosh \left(\alpha x^{2}\right)+\frac{\sqrt{\pi}}{2 \alpha^{\frac{3}{2}}}\left(x^{2}-\frac{3}{2 \alpha}\right) e^{\alpha x^{2}} \operatorname{erf}(\sqrt{\alpha} x)\right)\right],
\end{gathered}
$$

where $x=\sqrt{m_{0}^{2}(r) / E_{P}^{2}}$ and where we have used expression (41). The asymptotic expansion for large $x$ is

$$
\frac{\Lambda}{8 \pi G} \simeq-\frac{\left(2 \beta \alpha^{3 / 2}+\sqrt{\pi} \alpha^{2}\right) x}{4 \alpha^{7 / 2}}-\frac{8 \beta \alpha^{5 / 2}+3 \sqrt{\pi} \alpha^{3}}{16 \alpha^{11 / 2} x}+\frac{3}{128} \frac{16 \beta \alpha^{7 / 2}+5 \sqrt{\pi} \alpha^{4}}{\alpha^{15 / 2} x^{3}}+O\left(x^{-4}\right),
$$

while for small $x$, one gets

$$
\frac{\Lambda}{8 \pi G} \simeq-\frac{4 \alpha^{5 / 2}+3 \sqrt{\pi} \beta \alpha^{2}}{4 \alpha^{9 / 2}}+O\left(x^{3}\right) .
$$

If we set

$$
\beta=-\frac{\sqrt{\alpha \pi}}{2}
$$

then the linear divergent term of the asymptotic expansion (62) disappears and Eq. (61) vanishes for large $x$, while for small $x$ we get

$$
\frac{\Lambda}{8 \pi G} \simeq \frac{3 \pi-8}{8 \alpha^{2}}+O\left(x^{3}\right)
$$

where we have used the result of expansion (63). It is possible to show that with choice (64), the induced cosmological constant is always positive. 


\section{CONCLUSIONS}

In this contribution we have discussed how modifications of some basic points of General Relativity lead to finite contributions of ZPE without invoking neither a regularization nor a renormalization procedure. In particular, we have considered the effect that a NCG has on the counting of states with a deep modification of the measure in phase space. Such a modification introduces a Gaussian damping with a natural length: the Noncommutative length $\theta$. The final result is a finite ZPE, interpreted as a Cosmological Constant, regular at each point of the spacetime. On the other hand we have MDR's which provide an alternative way to keep under control UV divergences[6]. The application of MDR's is done in terms of a "rainbow metric" which, unfortunately, predicts a negative cosmological constant when one considers the same damping factor suggested from NCG. Nevertheless, by introducing appropriate variations of the original Gaussian proposal, positive contributions can be obtained.

\section{REFERENCES}

1. P. Hǒrava, JHEP, 0903, 020 (2009). ArXiv:0812.4287 [hep-th];P. Hǒrava, Phys. Rev. Lett. 102, 161301 (2009) ArXiv:0902.3657 [hep-th].

2. E.M. Lifshitz, Zh. Eksp. Toer. Fiz. 11, 255; 269 (1941).

3. P. Hǒrava and C. M. Melby-Thompson, Phys. Rev. D 82064027 (2010) ArXiv: 1007.2410 [hep-th].

4. P. Hǒrava, Phys. Rev. D 79, 084008 (2009) ArXiv: 0901.3775 [hep-th].

5. B. S. DeWitt, Phys. Rev. 160, 1113 (1967).

6. R. Garattini, Phys.Lett. B 685329 (2010) [arXiv:0902.3927 [gr-qc]].

7. R.Garattini, J. Phys. A 39, 6393 (2006); gr-qc/0510061. R. Garattini, J.Phys.Conf.Ser. 33, 215 (2006); gr-qc/0510062.

8. S. Capozziello and R. Garattini, Class.Quant.Grav. 24, 1627 (2007); gr-qc/0702075.

9. D.V. Vassilevich, Int. J. Mod. Phys. A 8, 1637 (1993).

10. M. Berger and D. Ebin, J. Diff. Geom. 3, 379 (1969). J. W. York Jr., J. Math. Phys., 14, 4 (1973); Ann. Inst. Henri Poincaré A 21, 319 (1974). P. O. Mazur and E. Mottola, Nucl. Phys. B 341, 187 (1990).

11. E. Elizalde, L. Vanzo and S. Zerbini, Commun.Math.Phys. 194, 613 (1998), hep-th/9701060; E. Elizalde, A. Filippi, L. Vanzo and S. Zerbini, Phys. Rev. D 57, 7430 (1998), hep-th/9710171.

12. T. Regge and J. A. Wheeler, Phys. Rev. 108, 1063 (1957).

13. G. 't Hooft, Nucl. Phys. B 256, 727 (1985).

14. P.A. Griffin and D.A. Kosower, Phys. Lett. B 233, 295 (1989).

15. R. Garattini, TSPU Vestnik 44 N7, 72 (2004); gr-qc/0409016.

16. R. Garattini and P. Nicolini, A noncommutative approach to the cosmological constant problem. e-Print: arXiv:1006.5418 [gr-qc].

17. G. Amelino-Camelia, Int.J.Mod.Phys. D 11, 35 (2002); gr-qc/0012051. G. Amelino-Camelia, Phys.Lett. B 510, 255 (2001); hep-th/0012238.

18. J. Magueijo and L. Smolin, Class. Quant. Grav. 21, 1725 (2004) [arXiv:gr-qc/0305055].

19. R. Garattini and G.Mandanici, in preparation. 